\title{
Investigation of The Relationship Between Asprosin Levels and Metabolic Parameters Observed in Clinical Follow-Up in Patients with Type 2 Diabetes
}

\author{
Ahmet Çagdaş Acara ${ }^{1 \oplus}$, Aslı Güler ${ }^{2}$ \\ 'Emergency Medicine Clinic, Urla State Hospital, İzmir, Turkey \\ ${ }^{2}$ Department of Family Medicine, University of Health Sciences, İzmir Bozyaka Training and Research Hospital, İzmir, Turkey \\ Address for Correspondence: Ahmet Çağdaş Acara, E-mail: cagdasacara@gmail.com \\ Received: 12.06.2019; Accepted: 19.06.2019; Available Online Date: 21.08.2019 \\ CCopyright 2018 by Dokuz Eylül University, Institute of Health Sciences - Available online at www.jbachs.org \\ Cite this article as: Acara AÇ, Güler A. Investigation of The Relationship Between Asprosin Levels and Metabolic Parameters Observed in Clinical Follow-Up in Patients with Type 2 \\ Diabetes. J Basic Clin Health Sci 2019; 3:128-133.
}

\begin{abstract}
Objectives: Asprosin is a newly identified adipokine which is involved in glucose and lipid metabolisms as well as inflammation. Type 2 diabetes mellitus (T2DM) associated with dysfunctions in glucose and lipid metabolisms is known as a metabolic disorder. The aim of this study was to investigate whether asprosin levels were changed in subjects with newly diagnosed T2DM (nT2DM) comparing to subjects with normal glucose tolerance (NGT) and to determine if asprosin levels were linked to metabolic parameters.

Method: This case control study included 68 subjects with nT2DM and 68 NGT age-, body mass index (BMI)- and gender-matched subjects. We used ELISA method for measuring the circulating asprosin levels. A 2-h 75-g oral glucose tolerance test was used for diagnosing of T2DM. Metabolic parameters of enrolled subjects were determined.

Results: Circulating asprosin levels were elevated in nT2DM subjects when compared to controls. (6.75 $\pm 1.54 \mathrm{vs.} 4.05 \pm 1.28 \mathrm{ng} / \mathrm{ml}, \mathrm{P}<0.001)$. Moreover, asprosin levels were found to be higher in nT2DM subjects with metabolic syndrome comparing to those without metabolic syndrome. Correlation analysis revealed that asprosin levels positively correlated with insulin, fasting blood glucose, insulin resistance and triglycerides whereas it displayed negative correlation with HDL cholesterol in only nT2DM subjects. Linear regression analyses showed an independent association between asprosin and insulin resistance. According to the logistic regression analyses, the subjects with the highest asprosin levels increased the probability of nT2DM prevalence.
\end{abstract}

Conclusions: Increased asprosin levels associated with insulin resistance and unfavorable outcomes of metabolic parameters in subjects with nT2DM.

Keywords: Type 2 diabetes mellitus, asprosin, insulin resistance, metabolic syndrome

\section{INTRODUCTION}

A novel peptide hormone namely asprosin is described as an important role player in glucose metabolism and it is secreted from white adipose tissue. It stimulates glucose secretion from liver by triggering $G$ protein-cAMP-PKA pathway (1). It is implicated that asprosin has some orexigenic properties in regulation of food intake (2). Focusing on asprosin functions and its relationship with insulin resistance has become a beneficial way of treating many kinds of metabolic disorders related to insulin resistance. The relationships between asprosin levels and metabolic disorders have been investigated to date (3-5). Asprosin levels were in positive association with insulin resistance in both humans and mice (3). In addition, asprosin induces inflammation and apoptosis of $\beta$ cells in pancreas (6). Elevated asprosin levels were found to be associated with insulin resistance in type 2 diabetes (T2DM) and polycystic ovary syndrome (PCOS) (3,5). Moreover, relation of asprosin levels with severity of coronary artery disease in subjects having unstable angina pectoris was described (4). Furthermore, asprosin has inflammatory effects (6).

Type 2 diabetes mellitus (T2DM) is a metabolic disorder associated with low-grade chronic inflammation. Insulin resistance plays a vital role in the developing of T2DM. Insulin secretion is increased as a response of insulin resistance and eventually insulin deficiency is an outcome of this process (7-11). Due to lacking of sufficient data regarding to the relation of asprosin with T2DM disorder, we aimed to focus on discovering the level of asprosin alteration in newly diagnosed T2DM (nT2DM). Moreover, we aimed to find whether there was a link between asprosin and metabolic parameters in $\mathrm{nT2DM}$. 


\section{MATERIALS and METHODS}

\section{Ethics statement}

We obtained the approval of the current study from ethics committee of Health Science University, Bozyaka Training and Research Hospital, Izmir, Turkey. We got the written and signed informed consent from each joined subject. We followed the principles in Declaration of Helsinki (revised in 2008) for each step of the study.

\section{Study design for subjects}

We planned the current study as a case-control study. We considered 312 subjects to choose the study test subjects. We enrolled 68 nT2DM and 68 age- and body mass index (BMI)matched subjects with NGT into the study consecutively. The range of enrolled subject's age was between $30-65$ years old. The study took for almost 6 months starting from June 2018 to January 2019 in Family Medicine of Bozyaka Training and Research Hospital of Izmir province in Turkey. The joined subjects did not consume alcohol and tobacco. A single specialist was responsible for scanning the detailed history and physical examination of enrolled subjects. A standard $75 \mathrm{~g}$ 2-h oral glucose tolerance test (OGTT) was completed for all subjects. Glucose tolerance of recruited subjects were defined based on ADA 2017 criteria as follows; normal glucose tolerance was explained as a fasting blood glucose (FBG) of $<100 \mathrm{mg} / \mathrm{dl}$ and/or a 2-h plasma glucose concentration of $<140 \mathrm{mg} / \mathrm{dl}$ and/or a HbA1C value of $<5.6 \%$ while prediabetes was defined as a FBG of 100-125 mg/dl (impaired fasting glucose), and/or a 2 -h plasma glucose concentration of 140-199 mg/dl (impaired glucose tolerance), and/or a HbA1c value of 5.7-6.4\%. Diabetes was defined as a FBG of $\geq 126 \mathrm{mg} / \mathrm{dL}$, and/or a 2-h plasma glucose concentration of $\geq 200 \mathrm{mg} / \mathrm{dl}$, and/ or a $\mathrm{HbA} 1 \mathrm{c}$ value of $\geq 6.5 \%$ (12). Metabolic syndrome was defined according to the National Heart, Lung, and Blood Institute/ American Heart Association criteria. The presence of metabolic syndrome is determined as having 3 or more of the following 5 criteria: 1) central obesity (waist circumference $\geq 102 \mathrm{~cm}$ for men and $\geq 88 \mathrm{~cm}$ for women), 2) increased triglyceride levels ( $\geq 150 \mathrm{mg} /$ dl) or specific medication for this lipid abnormality, 3) decreased HDL-cholesterol levels (<40 mg/dl for men and $<50 \mathrm{mg} / \mathrm{dl}$ for women) or specific medication for this lipid abnormality, 4) systemic hypertension (blood pressure $\geq 130 / 85 \mathrm{~mm} \mathrm{Hg}$ ) or antihypertensive therapy, and 5) increased FBG ( $\geq 100 \mathrm{mg} / \mathrm{dl})$ or history of diabetes mellitus or taking antidiabetic treatments (13).

\section{Exclusion criteria}

There were some criteria for excluding subjects from the current study such as being type 1 diabetic or gestational diabetes or being prediabetic patients, having the case of pregnancy or having breastfeeding or taking oral contraceptive pill, using carbohydrate metabolism-regulating/anti-obesity/anti-inflammatory drugs, using corticosteroid, using anti-lipemic and anti-hypertensive drugs, having cardiovascular disorder, having liver and renal dysfunctions and hypo-/hyperthyroidism, having malignant disorder history, suffering from connective tissue disease, having acute or chronic systemic disease and inflammatory illness. In addition, subjects with acute and chronic complications were excluded from the study.

\section{Anthropometric evaluation}

The standards of enrolled subjects including anthropometric measurements, ages (years), weight (kilogram), height (centimeter) and waist circumference (centimeter) were measured while subjects were barefoot and wearing casual clothes. We measured waist circumference between the lower rib margin and the iliac crest, the end of a gentle expiration' midway. Following a 15-minute resting period, we also measured the blood pressure of the subjects as they were in sitting position. We considered the following formula for calculation of BMI value: weight (kg)/square meter of height $(\mathrm{m} 2)$.

\section{Biochemical evaluation}

After at least a 10-hour fasting term we obtained the blood samples form antecubital veins in the morning (between 08:00-09:00). We put the gathered blood samples to in room temperature for at least 30 minutes to be clotted. Then, we centrifuged the clotted samples for 15 minutes at $2000 \mathrm{rpm}$ to obtain proper separation. The separated serum samples were kept in aliquots at $-80^{\circ} \mathrm{C}$ to evaluate asprosin levels. We also measured other parameters including fasting blood glucose (FBG) \& 2-h plasma glucose, glycated hemoglobin A1C (HbA1C), total cholesterol, serum insulin, high density lipoprotein cholesterol (HDL-C), triglycerides, high-sensitivity C-reactive protein (hs-CRP), creatinine, blood urea nitrogen (BUN), alanine transaminase (ALT) and aspartate transaminase (AST). We obtained some dedicated kits (Beckman Coulter Inc., CA, USA) with an auto-analyzer (Olympus AU 2700 Beckman Coulter Inc., CA, USA) to evaluate the circulating levels of FBG and 2-h plasma glucose, serum hs-CRP, total cholesterol, triglyceride, HDL-C, BUN, creatinine, ALT and AST of test subjects. The calculation of LDL-C was done via the Friedewald equation (LDL-C=total cholesterol-(HDL-C+Triglyceride/5)). Additionally, insulin levels of the serum were measured using Chemiluminescent microparticle immunoassay (CMIA) with its dedicated kits (Beckman Coulter Inc., CA, USA) as well as an auto-analyzer, UniCel DxI 800, Beckman Coulter Inc., CA, USA. We used high performance liquid chromatography (Variant II Turbo, Bio-Rad, CA, USA) to measure the levels of $\mathrm{HbA} 1 \mathrm{C}$. We tested insulin resistance of each sample via homeostasis model assessment of insulin resistance: HOMA-IR = fasting insulin $(\mu \mathrm{U} / \mathrm{ml}) \times$ fasting glucose $(\mathrm{mg} / \mathrm{dL}) / 405(14)$.

\section{Measurement of circulating asprosin levels}

The serum asprosin levels measured (in duplicate) by following instructions of manufactures as commercially available human ELISA kits were used (catalog number abx257694, Abbexa Ltd., Cambridge Science Park, Cambridge, UK). The values for the intraassay coefficient of variability (CV) and the inter-assay CV were $<6 \%$ and $<8 \%$ respectively. According to the manufacturer, the detecting range for asprosin is between $0.156 \mathrm{pg} / \mathrm{ml}$ and $10 \mathrm{ng} / \mathrm{ml}$.

\section{Statistical analysis}

We used the Statistical Package for the Social Sciences software version 18.0 (SPSS Inc. Chicago, IL, USA) for analyzing all data. We considered Kolmogorov-Smirnov test to determine distribution in each groups' variables. Normally distributed numeric variables are shown as mean \pm standard deviation (SD). Additionally, we used a student t-test for calculation of the presence of any difference between demographic and laboratory characteristic of both 
groups. We separated nT2DM subjects into two groups according to having metabolic syndrome status. Next we compared circulating asprosin levels between nT2DM subjects with and without metabolic syndrome using a student t-test. We considered Pearson's correlation coefficients to discover the link asprosin with other metabolic parameters. Apart from this, we tried to discover the prevalence of any independent association between asprosin and correlated parameters using a multiple regression model. The correlated variables were put into the model after multicollinearity test recognition (showing variance inflation factor (VIF) under 2.5). Then we tried to find any present association between asprosin levels (tertile) and having T2DM. We used multivariate logistic regression analysis for calculation odds ratio (OR) value. We adjusted the model by adding potential confounders such as age, BMI, HOMA$\mathrm{IR}$, hs-CRP and lipid parameters into the model. We analyzed the compatibility of the model using Hosmer and Lemeshow test $(\mathrm{P}>$ 0.05). A value of $95 \%$ was used for confidence interval $(\mathrm{Cl})$. A two sided $P$ value $<0.05$ was considered as a statistically significant.

\section{RESULTS}

Clinical and laboratory characteristics of the study population The comparative results of clinical and laboratory parameters of nT2DM and NGT are displayed in Table 1.
We found that asprosin levels were significantly higher in nT2DM than those in controls $(6.75 \pm 1.54$ vs. $4.05 \pm 1.28 \mathrm{ng} / \mathrm{ml}, \mathrm{P}<0.001)$ (Figure 1). Next we compared circulating levels of asprosin in nT2DM subjects with and without metabolic syndrome; the results showed that circulating asprosin levels were notably elevated in nT2DM subjects with metabolic syndrome $(7.22 \pm 1.57 \mathrm{vs} .5 .95 \pm 1.61 \mathrm{ng} / \mathrm{ml}$,

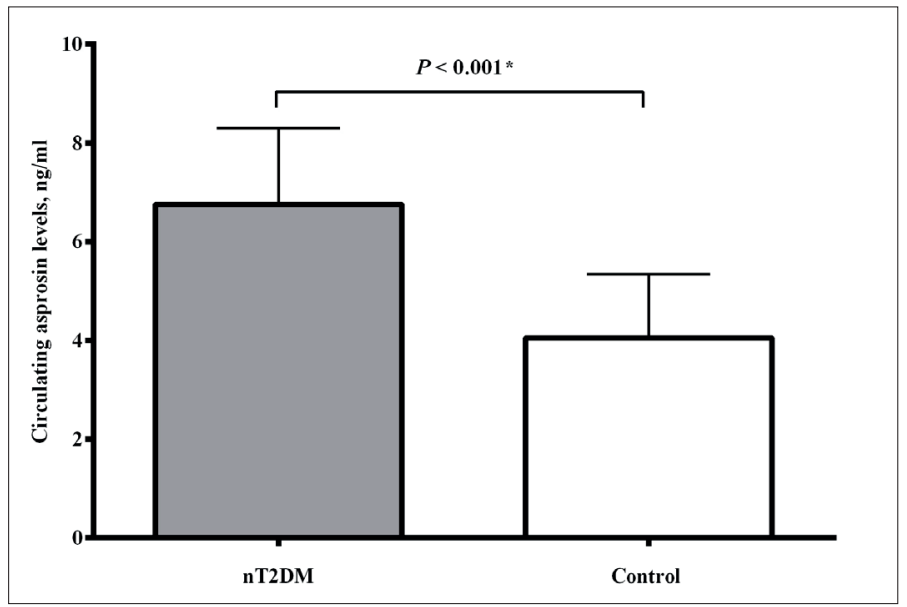

Figure 1. Circulating asprosin levels in both control and T2DM groups. A: $P$ value of $<0.05$ was considered as significant $\left({ }^{*}\right)$.

\begin{tabular}{|c|c|c|c|}
\hline Variables & $\begin{array}{c}\text { nT2DM } \\
n=68\end{array}$ & $\begin{array}{c}\text { Controls } \\
n=68\end{array}$ & $\mathbf{P a}$ \\
\hline Age, years & $50.61 \pm 8.77$ & $51.41 \pm 8.88$ & 0.601 \\
\hline Female/male & $34 / 34$ & $35 / 33$ & 0.864 \\
\hline $\mathrm{BMI}, \mathrm{kg} / \mathrm{m}^{2}$ & $26.89 \pm 4.30$ & $27.86 \pm 3.88$ & 0.173 \\
\hline $\mathrm{DBP}, \mathrm{mmHg}$ & $73.16 \pm 9.01$ & $71.83 \pm 8.80$ & 0.388 \\
\hline $\mathrm{FBG}, \mathrm{mg} / \mathrm{dl}$ & $126.70 \pm 22.75$ & $88.16 \pm 9.20$ & $<0.001^{*}$ \\
\hline 2-h OGTT, mg/dl & $228.45 \pm 34.21$ & $116.35 \pm 17.98$ & $<0.001^{*}$ \\
\hline $\mathrm{A} 1 \mathrm{c}, \%$ & $7.35 \pm 0.73$ & $5.34 \pm 0.25$ & $<0.001^{*}$ \\
\hline LDL-C, mg/dl & $162.92 \pm 32.45$ & $138.66 \pm 34.28$ & $<0.001^{*}$ \\
\hline HDL-C, mg/dl & $44.39 \pm 10.39$ & $49.95 \pm 13.62$ & $0.008^{*}$ \\
\hline Triglycerides, mg/dl & $206.27 \pm 66.80$ & $155.33 \pm 47.10$ & $<0.001^{*}$ \\
\hline $\mathrm{Hs}-\mathrm{CRP}, \mathrm{mg} / \mathrm{l}$ & $2.87 \pm 1.42$ & $1.26 \pm 0.59$ & $<0.001^{*}$ \\
\hline BUN, mg/dl & $25.10 \pm 6.04$ & $25.88 \pm 6.53$ & 0.474 \\
\hline Creatinine, mg/dl & $0.83 \pm 0.15$ & $0.87 \pm 0.21$ & 0.203 \\
\hline AST, IU/L & $21.27 \pm 11.08$ & $20.71 \pm 6.67$ & 0.721 \\
\hline ALT, IU/L & $24.75 \pm 11.43$ & $22.41 \pm 7.65$ & 0.162 \\
\hline
\end{tabular}

Results are given in mean \pm SD. a Independent t-test samples were used. A: $P$ value of $<0.05$ was considered as significant $\left({ }^{*}\right)$. A1 c: Glycosylated hemoglobin;

ALT: Alanine aminotransferase; AST: Aspartate aminotransferase; BMI: Body mass index; BUN: Blood urea nitrogen; DBP: Diastolic blood pressure; FBG: Fasting blood glucose;

HDL-C: High density lipoprotein cholesterol; HOMA-IR: Homeostasis model assessment of insulin resistance; Hs-CRP: High sensitivity C-reactive protein;

LDL-C: Low density lipoprotein cholesterol; SBP: Systolic blood pressure; 2-h OGTT: 2-hour oral glucose tolerance test; $\mathbf{n T 2 D M : ~ n e w l y ~ d i a g n o s e d ~ t y p e ~} 2$ diabetes mellitus. 


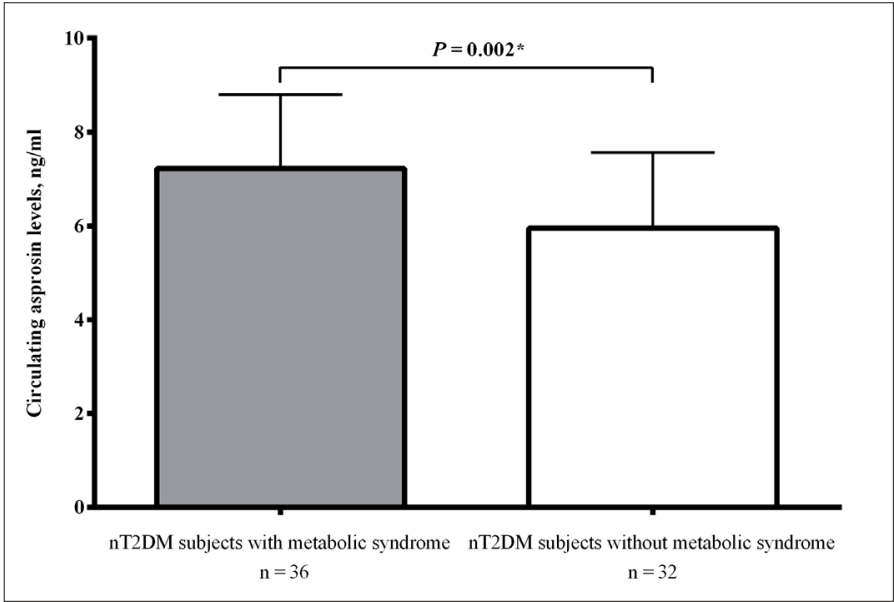

Figure 2. Circulating asprosin levels in nT2DM with and without metabolic syndrome groups. A: P value of $<0.05$ was considered as significant $(*)$.

$P=0.002$ ) (Figure 2). Insulin, insulin resistance marker HOMA-IR and inflammatory marker hs-CRP were found to be increased in nT2DM subjects compared to controls. Analyses of lipid profiles showed that total cholesterol ( $<200 \mathrm{mg} / \mathrm{dl})$, triglycerides $(<150 \mathrm{mg} / \mathrm{dl})$ and LDL-C (<100 mg/dl) were higher whereas HDL-C (>50 mg/dl) levels were lower in subjects with nT2DM comparing with controls.

\section{Correlation of asprosin with other parameters}

We used Pearson's correlation analyses to determine relation of asprosin with metabolic parameters in both nT2DM and control groups (Table 2).

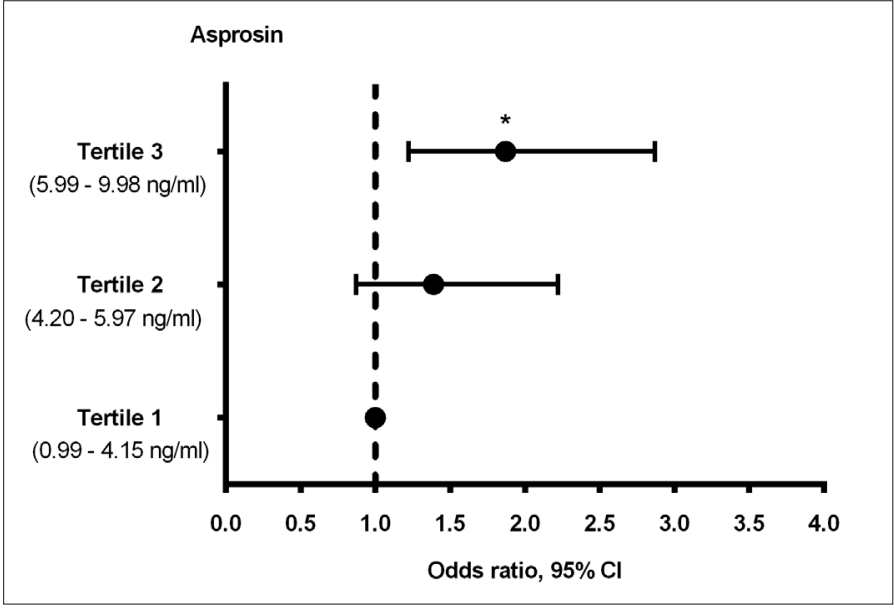

Figure 3. Association of asprosin with T2DM in adjusted models Multivariate adjusted OR for having T2DM according to the tertiles of UCN3 (reference, the lowest tertile). OR: Odds Ratio; Cl: Confidence Interval. A: P value of $<0.05$ was considered as significant ${ }^{*}$ ).

Circulating asprosin levels positively correlated with insulin, FBG, insulin resistance, hs-CRP levels in nT2DM subjects only. Besides, asprosin levels also displayed positive correlation with 2-h plasma glucose and $\mathrm{HbA} 1 \mathrm{C}$. We could find a positive correlation between asprosin and triglycerides whereas we discovered a negative correlation between HDL-C and asprosin levels in nT2DM only. We did not observe any correlation between asprosin levels and LDL-C. In both groups, asprosin levels showed a positive correlation with BMI and waist circumference.

Table 2. Correlation coefficient between asprosin levels and clinical parameters.

\begin{tabular}{|c|c|c|c|c|}
\hline & \multicolumn{4}{|c|}{ Asprosin } \\
\hline & \multicolumn{2}{|c|}{ nT2DM } & \multicolumn{2}{|c|}{ Control } \\
\hline & $\mathbf{r}$ & $\mathbf{P}$ & $\mathbf{r}$ & $\mathbf{P}$ \\
\hline Age & 0.011 & 0.379 & 0.023 & 0.456 \\
\hline SBP & 0.021 & 0.356 & 0.031 & 0.421 \\
\hline DBP & 0.062 & 0.335 & 0.056 & 0.327 \\
\hline BMI & 0.267 & $0.009^{*}$ & 0.213 & $0.011^{*}$ \\
\hline Waist circumference & 0.284 & $0.007^{*}$ & 0.224 & $0.010^{*}$ \\
\hline Insulin & 0.311 & $0.003^{*}$ & 0.112 & 0.055 \\
\hline FBG & 0.221 & $0.010^{*}$ & 0.109 & 0.057 \\
\hline 2-h OGTT & 0.118 & $0.033^{*}$ & 0.106 & 0.058 \\
\hline HOMA-IR & 0.278 & $0.005^{*}$ & 0.112 & 0.056 \\
\hline $\mathrm{A} 1 \mathrm{C}$ & 0.104 & $0.044^{*}$ & 0.076 & 0.088 \\
\hline $\mathrm{Hs}-\mathrm{CRP}$ & 0.215 & $0.0021^{*}$ & 0.103 & 0.076 \\
\hline Total cholesterol & 0.118 & 0.066 & 0.094 & 0.098 \\
\hline LDL-C & 0.103 & 0.088 & 0.081 & 0.124 \\
\hline HDL-C & -0.105 & $0.041^{*}$ & -0.053 & 0.076 \\
\hline Triglycerides & 0.121 & $0.031^{*}$ & 0.098 & 0.059 \\
\hline
\end{tabular}

Pearson's correlation analysis was used. r: Pearson's correlation coefficient. A: P value of $<0.05$ was considered as significant $(*)$. A1 c: Glycosylated hemoglobin;

BMI: Body mass index; DBP: Diastolic blood pressure; FBG: Fasting blood glucose; HDL-C: High density lipoprotein cholesterol; HOMA-IR: Homeostasis model assessment of insulin resistance; Hs-CRP: High sensitivity C-reactive protein; LDL-C: Low density lipoprotein cholesterol; SBP: Systolic blood pressure; 2-h OGTT: 2-hour oral glucose tolerance test; nT2DM: newly diagnosed type 2 diabetes mellitus. 


\begin{tabular}{|c|c|c|c|c|}
\hline & $\beta$ & & & $\mathbf{P}$ \\
\hline & & & & \\
\hline BMI & 0.865 & 0.298 & 1.432 & $0.015^{*}$ \\
\hline HOMA-IR & 1.132 & 0.764 & 1.501 & $0.009^{*}$ \\
\hline $\mathrm{Hs}-\mathrm{CRP}$ & 1.275 & 0.543 & 2.007 & $0.031^{*}$ \\
\hline 2-h OGTT & 0.912 & 0.104 & 1.721 & $0.046^{*}$ \\
\hline HDL-C & -0.092 & -0.101 & 0.203 & 0.078 \\
\hline $\mathrm{HbA} 1 \mathrm{C}$ & 0.421 & 0.108 & 0.734 & $0.044^{*}$ \\
\hline
\end{tabular}

Multiple linear regression analysis was used. $\beta$ : Unstandardized regression coefficient; Cl: Confidence interval; A P value of $<0.05$ was considered significant $(*)$.

BMI: Body mass index; HDL-C: High density lipoprotein cholesterol; HOMA-IR: Homeostasis model assessment of insulin resistance; LDL-C: Low density lipoprotein cholesterol; 2-h OGTT: 2-hour oral glucose tolerance test.

\section{Multivariate regression analysis}

To find independent correlation between circulating asprosin levels and correlated parameters (Table 3), multiple linear regression analysis was used; and results exhibited that asprosin levels were independently correlated with BMI, hs-CRP, 2-h plasma glucose, HbA1C, triglycerides and HOMA-IR. The correlation of asprosin with $\mathrm{HDL}-\mathrm{C}$ was vanished in this model.

\section{Multivariate Binary Logistic Regression Analysis}

To calculate OR for discovering the presence of any association between asprosin levels (tertile) and having nT2DM, we used a binary logistic regression analysis (Figure 3). The model was properly adjusted with potential confounders such as BMI, hs-CRP and lipids.

The highest tertile of asprosin levels associated with highest risk of nT2DM occurrence as compared with the lowest tertile asprosin $\left(\mathrm{OR}=1.87,95 \% \mathrm{Cl}=1.22-2.87, \mathrm{P}=0.033^{*}\right)$.

\section{DISCUSSION}

The present study displayed that circulating levels of asprosin were significantly higher in subjects with nT2DM than those subjects in controls. Circulating asprosin levels were also elevated in nT2DM subjects with metabolic syndrome compared to those nT2DM subjects without metabolic syndrome. Asprosin levels showed an independent association with insulin resistance, hs-CRP, 2-h plasma glucose, $\mathrm{HbA} 1 \mathrm{C}$, triglycerides and BMI. Moreover, the risk of having nT2DM was found to be higher in subjects having the highest levels of asprosin when compared to those subjects having lowest levels of asprosin.

The pathophysiology of T2DM is not clearly understood although it is implicated that insulin resistance plays a crucial role for the development of T2DM. Initially, insulin secretion is increased in parallel with the existence of insulin resistance $(8,9,11)$. Therefore, the molecules affecting the levels of insulin resistance and insulin secretion have been under investigation to date. Asprosin which is a newly discovered adipokine is able to play vital roles in glucose metabolism albeit contrary results have been reported regarding to this issue (15). In a preclinical study over mouse pancreatic $\beta$-cell line and human primary islets, asprosin application caused increased levels of inflammation as well as induced levels of apoptosis in $\beta$-cells in a dose-dependent manner (6). On the other hand, administration of asprosin resulted to hyperinsulinemia and hyperglycemia in animal models. In the same study, Romere et al. showed that insulin sensitivity was increased following blockage of asprosin (1). In a clinical study, asprosin levels were investigated as compared among 52 subjects with NGT, 40 subjects with impaired glucose regulation and 51 subjects with nT2DM. The circulating levels of asprosin were observed to be elevated in both subjects with impaired glucose regulation and nT2DM compared to controls whereas circulating levels of asprosin did not show significant difference between subjects with impaired glucose regulation and nT2DM (3). They also reported that asprosin levels showed a positive correlation with insulin resistance, $\mathrm{HbA} 1 \mathrm{C}$, triglycerides and first phase insulin secretion. They could not observe any correlation between asprosin and hs-CRP. Consistent to our study, we found that circulating asprosin levels were significantly higher in nT2DM subjects compared to controls. Moreover, circulating asprosin levels were higher in nT2DM subjects with metabolic syndrome than those nT2DM subjects without metabolic syndrome. We also found independent associations between asprosin and insulin resistance, triglycerides, hs-CRP, 2-h plasma glucose, HbA1C and BMI. In addition, the risk of having nT2DM was increased in parallel with induced levels of asprosin. In light of these data, asprosin could have the potential of playing different roles in glucose metabolism. Determination of asprosin levels in human disorders have been investigated. In a clinical study, circulating levels of asprosin was investigated in PCOS disorder associated with insulin resistance. They reported that increased circulating levels of asprosin were in relation to insulin resistance in women with PCOS. They also reported that asprosin levels were positively correlated with hs-CRP (5). Moreover, in a clinical study, a positive link between severity of acute coronary syndrome and asprosin levels was defined (4). In another study, obese subjects showed higher circulating levels of asprosin compared to controls. In the same study, it was added that following bariatric surgery caused a significant reduction in asprosin levels (16). Consistently, we reported in the current study that BMI and asprosin levels were independently associated with each other. 
Diabetes mellitus is associated with dyslipidemia although the exact mechanism of dyslipidemia in T2DM disorder is uncertain. Diabetic subjects tend to have unfavorable lipid profiles (17). In the current study, we found that total cholesterol, LDL-C and triglycerides levels were significantly elevated whereas HDL-C levels were significantly decreased in nT2DM subjects compared to controls. Moreover, asprosin levels showed positive correlation with triglycerides while it displayed negative correlation with HDL-C in nT2DM subjects. Following adjustment with other correlated parameters, the positive link of asprosin with triglycerides remained significant although the relationship between asprosin and HDL-C was disappeared. But relation of asprosin and triglycerides was not found to be unique according to previous data. There was no correlation between asprosin and triglyceride in PCOS subjects (5).

We came across with numerous limitations in our study Firstly, we measured insulin resistance using HOMA-IR, an indirect and inferior measuring technique method of determining insulin resistance instead of clamp method which is an advanced technique for measurement. Secondly, diabetic period of subjects was not precisely elucidated. Finally, although the study was relatively small-sized research, enrolled subjects were drug naïve to prevent drug effects over interaction between asprosin and metabolic parameters.

In conclusion, circulating asprosin levels were elevated in nT2DM subjects compared to NGT. In addition, increased circulating levels of asprosin were associated with insulin resistance and unfavorable lipid profile in subjects with nT2DM. Increased circulating asprosin levels linked to higher prevalence of T2DM. Obtained results from the current study may open a new door to explore further knowledge regarding to the effect of asprosin over different metabolic parameters in subjects with T2DM.

Informed Consent: Written informed consent was obtained from patients who participated in this study.

Compliance with Ethical Standards: We obtained the approval of the current study from ethics committee of University of Health Sciences, İzmir Bozyaka Training and Research Hospital, İzmir, Turkey

Peer-review: Externally peer-reviewed

Author Contributions: Concept - AÇA; Design - AÇA, AG; Supervision - AG; Fundings - AG; Materials - AG; Data Collection and/or Processing - AÇA, AG; Analysis and/or Interpretation - AG; Literature Search - AÇA, AG; Writing Manuscript - AÇA, AG; Critical Review - AÇA, AG

Conflict of Interest: No conflict of interest was declared by the authors.

Financial Disclosure: The authors declared that this study has received no financial support.

\section{REFERENCES}

1. Romere C, Duerrschmid C, Bournat J, et al. Asprosin, a FastingInduced Glucogenic Protein Hormone. Cell 2016;165:566-579. [CrossRef]

2. Duerrschmid $C$, He $Y$, Wang $C$, et al. Asprosin is a centrally acting orexigenic hormone. Nat Med 2017;23:1444-1453. [CrossRef]

3. Zhang L, Chen C, Zhou N, Fu Y, Cheng X. Circulating asprosin concentrations are increased in type 2 diabetes mellitus and independently associated with fasting glucose and triglyceride. Clin Chim Acta 2019;489:183-188. [CrossRef]

4. Acara, A.C., Bolatkale, M., Kızıloğlu, I.., İbişoğlu, E., Can, Ç.: A novel biochemical marker for predicting the severity of ACS with unstable angina pectoris: Asprosin. Am J Emerg Med 2018; 36:1504-1505. [CrossRef]

5. Alan M, Gurlek B, Yilmaz A, et al. Asprosin: a novel peptide hormone related to insulin resistance in women with polycystic ovary syndrome. Gynecol Endocrinol 2018;35:220-223. [CrossRef]

6. Lee $\mathrm{T}$, Yun $\mathrm{S}$, Jeong JH, Jung TW. Asprosin impairs insulin secretion in response to glucose and viability through TLR4/JNK-mediated inflammation. Mol Cell Endocrinol 2019;486:96-104. [CrossRef]

7. Marshall BA, Ren JM, Johnson DW, et al. Germline manipulation of glucose homeostasis via alteration of glucose transporter levels in skeletal muscle. J Biol Chem 1993;268:18442-18445. Available at: [CrossRef]

8. De Fronzo RA, Tripathy D. Skeletal Muscle Insulin Resistance Is the Primary Defect in Type 2 Diabetes. Diabetes Care 2009;32:S157S163. [CrossRef]

9. Kahn BB. Type 2 diabetes: when insulin secretion fails to compensate for insulin resistance. Cell 1998;92:593-596. [CrossRef]

10. Ogurtsova K, da Rocha Fernandes JD, Huang $Y$, et al. IDF Diabetes Atlas: Global estimates for the prevalence of diabetes for 2015 and 2040. Diabetes Res Clin Pract 2017;128:40-50. [CrossRef]

11. Taylor R. Insulin Resistance and Type 2 Diabetes. Diabetes 2012;61:778-779. [CrossRef]

12. 2. Classification and Diagnosis of Diabetes. Diabetes Care. 40, S11S24 (2017).

13. Grundy SM, Cleeman JI, Daniels SR, et al. Diagnosis and management of the metabolic syndrome: an American Heart Association/National Heart, Lung, and Blood Institute Scientific Statement. Circulation 2005; 112:2735-52. [CrossRef]

14. Matthews, D.R., Hosker, J.P., Rudenski, A.S., Naylor, B.A., Treacher, D.F., Turner, R.C.: Homeostasis model assessment: insulin resistance and beta-cell function from fasting plasma glucose and insulin concentrations in man. Diabetologia. 28, 412-9 (1985).

15. Wang $\mathrm{Y}$, Qu H, Xiong X, et al. Plasma Asprosin Concentrations Are Increased in Individuals with Glucose Dysregulation and Correlated with Insulin Resistance and First-Phase Insulin Secretion. Mediators Inflamm 2018;9471583. [CrossRef]

16. Wang $\mathrm{C}-\mathrm{Y}$, Lin T-A, Liu K-H, et al. Serum asprosin levels and bariatric surgery outcomes in obese adults. Int J Obes 2018;43:1019-1025. [CrossRef]

17. Wu L, Parhofer KG. Diabetic dyslipidemia. Metabolism 2014;63:14691479. [CrossRef] 